\title{
ISCEV standard for clinical pattern electroretinography-2007 update
}

\author{
Graham E. Holder • Mitchell G. Brigell · Marko Hawlina · Thomas Meigen · \\ Vaegan - Michael Bach · for the International Society for Clinical Electrophysiology of Vision
}

Received: 28 February 2007/Accepted: 1 March 2007/Published online: 14 April 2007

(C) Springer Science+Business Media B.V. 2007

\begin{abstract}
The pattern electroretinogram (PERG) is a retinal response evoked by viewing a temporally alternating pattern, usually a black and white checkerboard or grating. The PERG is important in clinical and research applications because it provides information both about retinal ganglion cell function and, because the stimulus is customarily viewed with central fixation, the function of the macula. The PERG can therefore facilitate interpretation of an
\end{abstract}

This document is available on the ISCEV website $<w w w . i s c e v . o r g>$.

G. E. Holder $(\bowtie)$

Moorfields Eye Hospital, City Road,

London EC1V 2PD, UK

e-mail: graham.holder@moorfields.nhs.uk

M. G. Brigell

Pfizer Global R\&D, Ann Arbor, MI, USA

M. Hawlina

University Eye Hospital, Medical Centre Ljubljana, Ljubljana, Slovenia

T. Meigen

Department of Ophthalmology,

University of Würzburg, Würzburg, Germany

Vaegan

University of New South Wales, Sydney, Australia

M. Bach

University Eye Clinic, Freiburg, Germany abnormal pattern VEP by revealing the retinal responses to a similar stimulus to that used for the VEP. However, practitioners may have difficulty choosing between the different techniques for recording the PERG that have been described in the literature. The International Society for Clinical Electrophysiology of Vision published a standard for clinical PERG recording in 2000 to assist practitioners in obtaining good quality reliable responses and to facilitate inter-laboratory communication and comparison. This document is the scheduled revision of that standard.

Keywords Clinical electrophysiology ·

Electroretinogram · Pattern electroretinogram .

Retina $\cdot$ Macula

\section{Introduction}

The pattern electroretinogram (PERG) is a retinal biopotential evoked when a temporally modulated patterned stimulus (checkerboard or grating) of constant mean luminance is viewed. The PERG is most often evoked by alternating contrast-reversal of a black and white square checkerboard pattern with central fixation. It may be altered in dysfunction confined to the macula or to the retinal ganglion cells, which do not significantly affect the a- and b-waves of the conventional full-field ERG, and thus the PERG receives clinical and research attention in both 
neurological and ophthalmological practice. Clinically, the PERG can be used in a patient with an abnormal visual evoked potential to establish whether a retinal (macular) disorder is present, and thus differentiate between macular and optic nerve dysfunction as a cause for the VEP abnormality. It can also directly demonstrate retinal ganglion cell dysfunction.

However, the PERG is a very small signal, typically in the region of $2-8 \mu \mathrm{V}$ across a normal population, and PERG recording is technically more demanding than standard flash ERGs. The amplitude values recorded will depend upon stimulus characteristics and the electrodes being used. The recordings published in the literature vary considerably in technique and technical quality; this document is intended to guide new or existing users to the most appropriate techniques for recording a standard clinical PERG.

The International Society for Clinical Electrophysiology of Vision (ISCEV) initially published "PERG Guidelines" [1], intended as a guide to practice and to assist in interpretation of PERGs. Those guidelines were subsequently revised to a PERG standard [2] and the present document is the scheduled revision of that standard. The standard PERG represents a minimum protocol for recording a PERG with straightforward technical procedures that should allow reproducible responses to be recorded under defined conditions. As a minimum, it is intended that the standard method and responses be widely used, but in addition to rather than to the exclusion of other paradigms. Common additional PERG techniques are also briefly described in this document, and individual laboratories are encouraged to use additional stimuli and protocols tailored to their own requirements.

The standard is based upon equipment and analytic capabilities currently available in most neurophysiological or ophthalmological electrodiagnostic clinics. This document addresses recording conditions and technology specific to the PERG, and presumes that the reader already has basic understanding and skills in clinical electrophysiology. Although much of the document will apply equally to adults and children, the standard is not necessarily appropriate to paediatric applications. The standard will be reviewed by ISCEV in a further four years.

\section{Waveform nomenclature and measurement}

The waveform of the PERG evoked by patternreversal stimuli depends on the temporal frequency of the stimulus. By convention, positivity is displayed upward.

\section{Transient PERG}

The ISCEV standard PERG is a transient response i.e. a response that is effectively complete before the next contrast reversal. Transient recording allows separation of the PERG components. At low temporal frequencies ( $<6$ reversals per second (rev/s); equivalent to $<3 \mathrm{~Hz}$ ) transient PERGs are obtained (Fig. 1). The PERG waveform in normal subjects usually consists of a small initial negative component with a peak time of approximately $35 \mathrm{~ms}$, N35, which is followed at $45-60 \mathrm{~ms}$ by a much larger positive component (P50). This positive component is followed by a larger negative component at $90-100 \mathrm{~ms}$ (N95).

For the transient PERG, amplitude measurements are made between peaks and troughs: the P50 amplitude is measured from the trough of N35 to the peak of P50. In some patients the N35 is poorly defined; in these cases N35 is replaced by the average baseline between time zero and the onset of P50. The N95 amplitude is measured from the peak of P50 to the trough of N95. It should be recognised that measured in this way, N95 amplitude includes the P50 amplitude and P50 that of N35. It can be argued that baseline to peak measurements may be more meaningful. However, the P50 and N95 components may not be independent. At the time of writing there are no peer-reviewed data attempting to resolve these

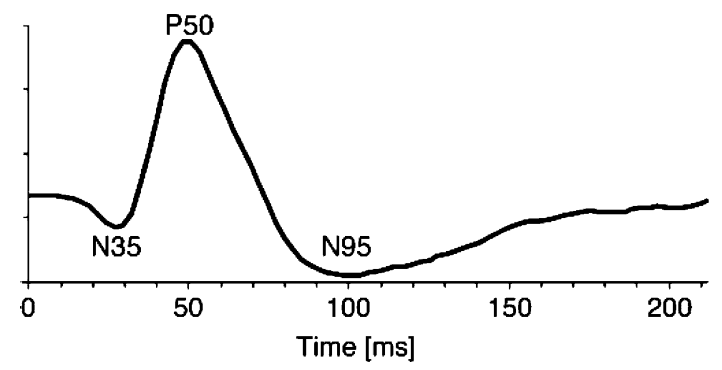

Fig. 1 A normal PERG. The amplitude of P50 in a normal subject is usually between 2.0 and $4.0 \mu \mathrm{V}$ 
issues, and given the wealth of data already published using peak-to-peak measures, they remain the standard measures for PERG amplitudes.

The times to peaks in the waveform (implicit time) should be measured from the onset of the contrastreversal to the peak of the component concerned; it should be noted that the highest absolute amplitude point on a waveform will not always be appropriate for the definition of the peak if there is contamination from muscle activity or other artifacts. The peak should be designated where it would appear on a smoothed or idealised waveform (see Fig. 1). The peak times or implicit times are often (erroneously) referred to as latencies. Correctly, latency refers to the delay prior to the onset of a response, not to the maximum activity of a peak.

\section{Basic technology}

Standard equipment for visual stimulus generation, amplification of physiological signals, and the recording and storing of electrophysiological data, is required for PERG testing. Information about the calibration of equipment and measurement of the parameters specified in this standard appears in the ISCEV Calibration Guidelines [3].

\section{Electrodes}

\section{Recording electrodes}

Clinical ERG electrodes that contact the cornea or nearby bulbar conjunctiva should be used as the active electrodes to record standard PERGs. Electrodes that degrade image quality on the retina (this includes all contact lens electrodes) must not be used. Thin conductive fibres and foils can usually be positioned without topical anaesthesia. Electrode integrity should be checked prior to insertion, to meet guidelines for each electrode type. It is recommended not to measure impedance in situ unless explicitly specified by the particular equipment manufacturer. Electrodes should be carefully positioned to minimise instability (a major source of artifact or interference). Those who perform the test should be aware of possible causes of artifact.

- Fibre electrodes are best positioned in relation to the upper margin of the lower eyelid. Some place the electrode in the lower conjunctival fornix (under the lower eyelid); such a position may reduce trial-to-trial variability but will also result in a lower amplitude PERG. Optimum stability is achieved by tethering the electrode at the nasal canthus.

- Foil electrodes should be positioned directly under the centre of the pupil so that there is minimal or no movement of the electrode when the patient blinks. This is best achieved by having the foil curve over the lower eyelashes without contacting them, and then tethering the lead to the cheek. The junction of the electrode and lead should form as straight a line as possible and the junction should not touch the skin.

- Loop electrodes should be hooked into the lower fornix. Loops should be folded so that the contact windows on otherwise insulated wire are positioned on the bulbar conjunctiva, about $5 \mathrm{~mm}$ under the limbus. Loop electrodes should not touch the cornea. To achieve this, the limbs of the loop should diverge widely (15-20 $\mathrm{mm}$ ) before entering the fornix. The lead is then taped to the cheek.

The appropriate techniques for individual electrode types are very important to achieve stable and reproducible PERG recordings. Additional sources should be consulted in relation to the specific electrode used.

Surface (skin) active electrodes should not routinely be used for recording the standard PERG; a surface electrode positioned on the lower eyelid will record PERGs of lower amplitude than those recorded from an electrode in contact with the eye. Surface electrode recordings may however be useful when a corneal electrode is contraindicated or in paediatric practice. The use of a surface electrode to record the PERG is a variation from the standard and should be noted in the report.

\section{Reference electrodes}

Separate surface reference electrodes should be placed on the skin near the ipsilateral outer canthus of each eye. Mastoid, earlobe or forehead locations may result in contamination of the PERG from cortical potentials or the fellow eye. If monocular PERG recording is performed, the electrode in the occluded eye may be used as a reference. 


\section{Ground electrodes}

A separate surface electrode should be attached and connected to the amplifier "ground input'; the forehead would be a typical location, but other locations are acceptable as the location of the ground electrode should not affect the standard PERG.

\section{Surface electrodes}

The skin should be prepared with a suitable cleaning agent, and a suitable conductive paste used to ensure good electrical connection. The impedance between the skin electrodes used for reference and ground, measured on the subject, should be less than $5 \mathrm{k} \Omega$. Since the electrode in the eye will have very low impedance, a low impedance of the reference electrode is also important to obtain recordings as free as possible from mains (line frequency) interference.

\section{Electrode cleaning and sterilisation}

Electrodes should be cleaned and sterilised according to local health and safety regulations. The ISCEV Standard for full-field flash ERGs describes the appropriate care of ERG electrodes [4].

Stimulus parameters

This standard specifies the protocol for basic clinical PERG recording. Laboratories may choose to test more conditions or parameters than are described herein.

\section{Field and check size}

A black and white reversing checkerboard should be used for the standard PERG. It is not necessary to use a square stimulus field, but the aspect ratio between the width and the height of the stimulus field should not exceed $4: 3$. The mean of the width and the height of the stimulus field should be $15^{\circ}\left( \pm 3^{\circ}\right)$ with a check size of $0.8^{\circ}\left( \pm 0.16^{\circ}\right)$.

\section{Luminance}

PERGs are difficult to record with low stimulus luminance, and a photopic luminance level for the white areas of greater than $80 \mathrm{~cd} \cdot \mathrm{m}^{-2}$ is required.
The mean luminance of the stimulus screen must be constant during checkerboard reversals (i.e. no transient luminance change).

\section{Contrast}

The contrast between black and white squares should be maximal (close to 100\%) for the standard PERG and not less than $80 \%$. The contrast and luminance used should be reported.

\section{Frame rate}

Raster-based CRTs are typically used to present the pattern stimuli. The frame rate of the CRT is a significant stimulus parameter for PERGs, and a frequency of $75 \mathrm{~Hz}$ or greater should be used.

\section{Background illumination}

The luminance of the background beyond the checkerboard field is not critical when using the standard PERG technique providing dim or ordinary room lighting is used; ambient lighting should be the same for all recordings. Care should be taken to keep bright lights out of the subjects' direct view. Pupil diameter should be recorded.

\section{Reversal rate}

The standard transient PERG should be obtained using a reversal rate of $4 \mathrm{rev} / \mathrm{s} \pm 0.8 \mathrm{rev} / \mathrm{s}$ (i.e. $2 \mathrm{~Hz} \pm 0.4 \mathrm{~Hz})$.

\section{Calibration}

All stimulus parameters including luminance and contrast should be calibrated either locally or by the manufacturer and regular recalibration is advised [3].

Recording equipment

\section{Amplification systems}

AC-coupled amplifiers with a minimum input impedance of $10 \mathrm{M} \Omega$ should be used. Amplification systems must be electrically isolated from the patient according to the current safety standards for medical 
recording systems. The frequency response of bandpass amplifiers should include the range from 1-100 Hz; analogue notch filters (that suppress signals at the alternating current line frequency) should not be used. Some users may encounter severe electromagnetic interference from the stimulus display that makes it difficult to obtain satisfactory recordings with these filter settings. Ideally, such interference should be eliminated by shielding or modifying the equipment; rearranging the electrode leads may be of benefit.

\section{Averaging and signal analysis}

Signal averaging is necessary because of the small amplitude of the PERG. The analysis period (sweep time) for the standard PERGs should be $150 \mathrm{~ms}$ or greater. Some laboratories assess baseline stability by displaying two successive responses on the same trace.

\section{Artefact rejection}

Computerised artifact rejection is essential. The limits for rejection should be set at no higher than $\pm 50 \mu \mathrm{V}$, and preferably lower. The amplifiers must return to baseline rapidly following artifactual signals to avoid inadvertent storage of non-physiological data.

\section{Sampling rate}

A minimum sampling rate of $1,000 \mathrm{~Hz}(1 \mathrm{~ms}$ per point) is recommended. See the calibration standard (3) for further information.

\section{Data display systems}

Display systems must have adequate resolution to represent accurately the characteristics of this small amplitude signal. Ideally the recording system provides simultaneous display of the input signal and the accumulating average. In the absence of a simultaneous display, the system should allow a rapid alternation between displaying the input signal and displaying the current average so that the quality of the input signal can be adequately monitored. Even with a computerised artifact rejection system, it is important that the input signal be continuously monitored for baseline stability and the absence of amplifier blocking.

\section{Clinical protocol}

Preparation of the patient

\section{Positioning}

The patient should be as comfortable as possible with their head in a stable position against a head-rest. A chin rest is inappropriate and should not be used.

\section{Pupils}

The PERG should be recorded without dilatation of the pupils, to preserve accommodation and thus retinal image quality.

\section{Fixation}

A fixation mark in the centre of the screen at a node of the checkerboard is essential. If there are any doubts about the quality of fixation in an individual patient, an effective method is to give the patient a (laser) pointer and have them point at the middle of the screen throughout. Excessive blinking during recording should be discouraged, pauses may be advantageous.

\section{Refraction}

Because of the nature of the stimulus, PERG examination should be performed with optimal visual acuity at the testing distance. Patients should wear the appropriate optical correction for the test distance.

\section{Monocular and binocular recording}

Proper positioning of recording and reference electrodes will permit either monocular or binocular recording of the PERG. Binocular recording is recommended for the standard PERG because it is generally more stable, it reduces examination time and it allows fixation by the better eye in cases of asymmetric visual loss. Monocular stimulation is required to record the PERG and the VEP simultaneously. 


\section{Recording}

A minimum of 100 artefact-free sweeps should be collected and averaged for a standard PERG. More sweeps, perhaps as many as 300 , will be needed when the PERG is small or undetectable or in a "noisy" subject. At least two trials for each stimulus condition should be obtained to confirm reproducibility (i.e. at least one replication). It may be beneficial to superimpose repeated PERG recordings to evaluate quality and reproducibility.

\section{PERG reporting}

\section{Reporting}

It is recommended that all reports contain measurements of P50 and N95 amplitude (see above), and P50 peak time (the peak of N95 is often rather broad precluding accurate peak time measurement of this component). All reports should also contain the stimulus parameters (luminance, contrast, and field size), and normal ranges for the laboratory concerned. Pupil size should be noted. Whenever practical, reporting of PERG results should include representative waveforms with appropriate amplitude and time calibrations, marks for the N35, P50 and N95 components, and should show replications.

\section{Normative data/reference ranges}

At present there are no standard international reference ranges for PERG measurements. Each laboratory should establish normal values for its own equipment and patient population. It should be noted that there are PERG changes with age.

\section{Additional tests}

\section{Large field PERGs}

For some applications, such as glaucoma assessment, a larger field, such as $30^{\circ}$, may be more appropriate. Further, some practitioners find the improved signalto-noise ratio obtained with a larger field may have advantages; users may wish to consider recording a large field PERG in addition to the standard response.
Steady-state PERG

At higher temporal frequencies, i.e. above $10 \mathrm{rev} / \mathrm{s}$ $(5 \mathrm{~Hz})$, the successive waveforms overlap and a "steady-state", PERG is evoked. There are situations in which the steady-state PERG is useful and some laboratories favour it for glaucoma studies. Since little extra time is required, laboratories may wish to consider recording it in addition to the transient response.

For steady-state PERG, a reversal rate of approximately $16 \mathrm{rev} / \mathrm{s}(8 \mathrm{~Hz})$ is recommended. The steady-state PERG waveform is roughly sinusoidal, and interpretation of steady-state PERGs requires measurement of amplitude and phase shift (relative to the stimulus) of the second harmonic by Fourier analysis. The presence of a significant first harmonic indicates technical problems. For correct interpretation the analysis period must be an integer number of stimulus cycles, preferably greater than 6. Steadystate PERG recording without the capability for such analysis is not recommended.

Reporting of steady-state PERGs should include the amplitude and phase shift of the PERG at the reversal rate (i.e. at the second harmonic of the stimulus rate in $\mathrm{Hz}$ ).

Acknowledgements This Standard forms part of series of Standards, Recommendations and Guidelines prepared by the International Society for Clinical Electrophysiology of Vision (ISCEV). Particular thanks are extended to John Robson and Daphne McCullough for their careful reading and comment.

\section{References}

1. Marmor MF, Holder GE, Porciatti V, Trick GL, Zrenner E (1996) Guidelines for basic pattern electroretinography. Recommendations by the International Society for Clinical Electrophysiology of Vision. Doc Ophthalmol 91:291-298

2. Bach M, Hawlina M, Holder GE, Marmor MF, Meigen T, Vaegan, Miyake Y (2000) Standard for Pattern Electroretinography. Doc Ophthalmol 101:11-18

3. Brigell M, Bach M, Barber C, Kawasaki K, Kooijman A (1998) Guidelines for calibration of stimulus and recording parameters used in clinical electrophysiology of vision. Doc Ophthalmol 95:1-14

4. Marmor MF, Holder GE, Seeliger MW, Yamamoto S (2004) Standard for clinical electroretinography (2004 update). Doc Ophthalmol 108:107-114 